\title{
DESIGN AND PROTOTYPE TESTS OF A LARGE-APERTURE 37-53 MHZ FERRITE-TUNED BOOSTER SYNCHROTRON CAVITY
}

\author{
Mark Champion, Tim Berenc, Mike May, John Reid, FNAL*, Batavia, IL 60510, USA
}

\section{Abstract}

The Booster synchrotron at Fermilab employs eighteen 37-53 MHz ferrite-tuned double-gap coaxial radiofrequency cavities for acceleration of protons from $400 \mathrm{MeV}$ to $8 \mathrm{GeV}$. The cavities have an aperture of 2.25 inches and operate at $55 \mathrm{kV}$ per cavity. Future high duty factor operation of the Booster will be problematic due to unavoidable beam loss at the cavities resulting in excessive activation. The power amplifiers, high maintenance items, are mounted directly to the cavities in the tunnel. A proposed replacement for the Booster, the Proton Driver, will utilize the Booster radiofrequency cavities and requires not only a larger aperture, but also higher voltage. A research and development program is underway at Fermilab to modify the Booster cavities to provide a 5 -inch aperture and a $20 \%$ voltage increase. A prototype has been constructed and high power tests have been completed. The cavity design and test results will be presented.

\section{INTRODUCTION}

The Booster synchrotron at Fermilab accelerates protons from $400 \mathrm{MeV}$ to $8 \mathrm{GeV}$ with a cycle time of $67 \mathrm{~ms}$ and a repetition rate of a few Hertz. The machine provides proton beams with intensities up to $5 \mathrm{e} 12$ protons per cycle (6e10 protons/bunch x 84 bunches). Future fixed target experiments require higher duty factor operation of the Booster, and longer range physics plans suggest the construction of a Proton Driver [1], a high intensity Booster replacement. In light of these plans, a proposal [2] was made to modify an existing Booster rf cavity to achieve two goals: (1) to enlarge the beam aperture from 2.25 to 5 inches; (2) to increase the cavity voltage by $20 \%$ from $55 \mathrm{kV}$ to $66 \mathrm{kV}$. Aperture enlargement for the existing Booster synchrotron will result in less activation of the rf cavities and power amplifiers, thereby permitting required regular maintenance during future high duty factor operation. Increased cavity voltage will allow for operation with fewer than $100 \%$ of installed cavities. Consequently downtime due to unscheduled maintenance will be reduced. Aperture enlargement for the Proton Driver is necessary to accommodate the higher beam intensity, whereas the increased cavity voltage is required to increase the beam energy without increasing the acceleration cycle time.

\section{OVERVIEW OF EXISTING CAVITY}

The Booster depends on $18 \mathrm{rf}$ cavities for acceleration, each cavity providing approximately $55 \mathrm{kV}$ over a

\footnotetext{
* Fermilab is operated by the Universities Research Association under contract to the U.S. Department of Energy.
}

frequency of 37 to $53 \mathrm{MHz}$. Hence a ring voltage of $990 \mathrm{kV}$ is achieved. The rf cavity is a center-fed coaxial double-gap structure with an electrical length of 136 degrees, gap-to-gap, as illustrated in Figure 1. The $150 \mathrm{~kW}$ power amplifier is mounted directly to the cavity and is coupled to the center conductor via a $1200 \mathrm{pF}$ anode DC blocking capacitor. The cavity frequency is controlled via three parallel ferrite-loaded inductive tuners attached at the center of the cavity. The bias windings are series connected and driven by a $2500 \mathrm{~A}$ programmable bias supply. Two 12-inch diameter conical coaxial aluminum oxide windows separate the beam vacuum from the air-filled center section of the cavity. The gap spacing is 0.9 inches and the beam aperture is 2.25 inches. The cavity anode to gap step-up ratio varies from 1.2 to 1.45 over the frequency range 37 to $53 \mathrm{MHz}$.

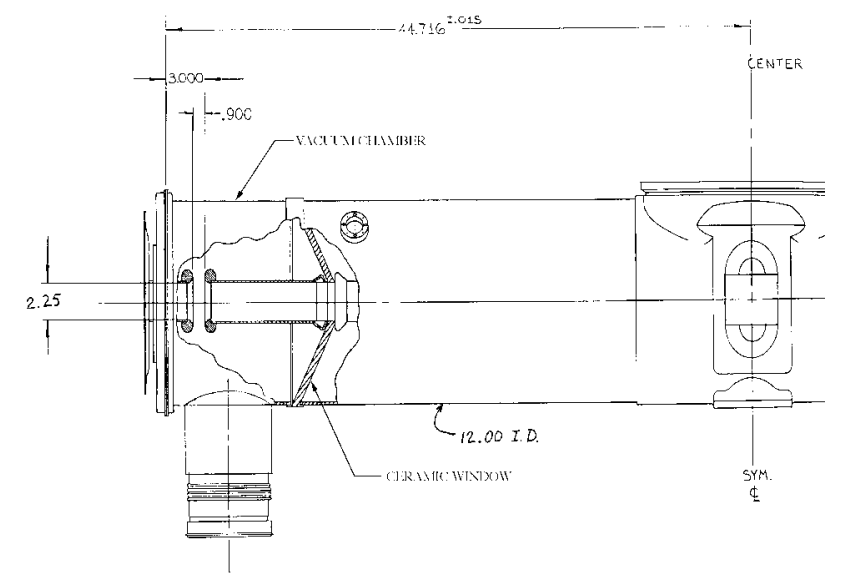

Figure 1. Detail of Booster cavity gap geometry.

\section{APERTURE ENLARGEMENT AND TUNING}

A damaged Booster rf cavity has been the basis of the prototype work to date. Both ceramic windows, one of which was already broken, were removed to allow for extraction of the cavity drift tube. The center conductor was cut off along the taper on both sides of the center hub and new 5.5 inch outside diameter ends were attached along with variable spacing gap electrodes. The 5.5 inch outside diameter was chosen to allow for an inside diameter of 5 inches. The 1/4 inch clearance is needed because the drift tube is made up of three concentric tubes: a copper beam tube, a high permeability magnetic shielding tube, and a copper inner conductor. The end plates of the cavity were modified to match the 5 inch aperture. The gap spacing is variable up to a maximum of 3 inches on the prototype cavity. Aluminum oxide 
ceramic washers were purchased to approximate the capacitive loading that will be present in a new vacuum window design. As a first step, two standard Booster cavity ferrite tuners were mounted on the prototype and measurements were performed with a special cut-away power amplifier that allows access to the anode via the screen basket. The impedance at the anode of the power tube can be measured by inserting the probe of a vector voltmeter into the screen basket between the anode and screen. The ferrite tuner bias current is set and the frequency of the vector voltmeter is adjusted until a phase angle of zero is indicated. Following this procedure, curves of anode impedance versus resonant frequency may be measured for various configurations.

Three tuner configurations were explored: two Booster tuners, three Booster tuners, and two Booster tuners with one Main Injector tuner. The resulting anode impedance versus cavity frequency curves are shown in Figure 2. The data for a standard Booster cavity is included for comparison. Clearly the lower inductance of the Main Injector tuner is necessary to make up for the increased cavity aperture. Two different gap spacings were investigated: 0.9 inch and 2 inch. The larger gap spacing results in a slightly higher upper frequency limit due to the decrease in gap capacitance. These data do not extend to higher frequencies because the measurements were performed using only a 1000 A bias supply.

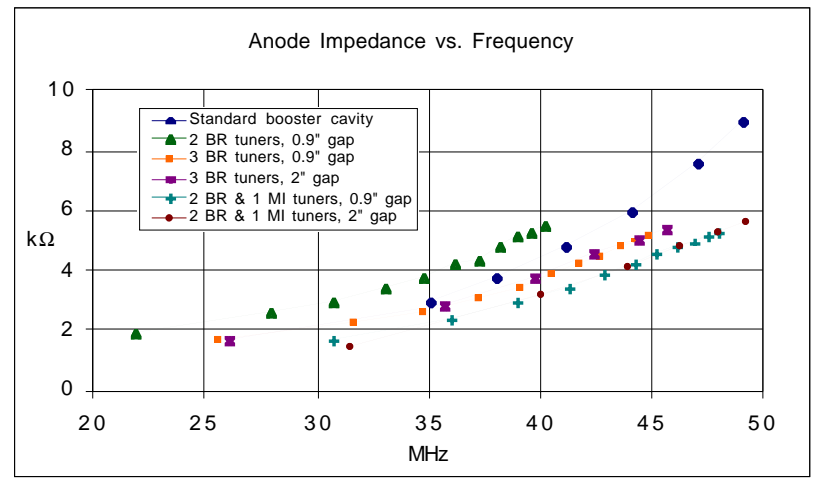

Figure 2. Curves of prototype cavity anode impedance for various tuner and gap configurations.

Based on the preceding results, the next step was to install the prototype cavity configured with two Booster tuners and one Main Injector tuner at the MI-60 test station where tests with a $2500 \mathrm{~A}$ bias supply were performed. Bias currents from 0 to $2500 \mathrm{~A}$ provided a frequency tuning range from 29.8 to $53.4 \mathrm{MHz}$ with a gap spacing of 0.9 inches and no dielectric loading (Figure 3). A standard Booster cavity has a maximum frequency of $53.3 \mathrm{MHz}$ for comparison. Aluminum oxide windows installed near the gaps required opening up the gap spacing to 1.9 inches to reach an upper frequency of 53.1 MHz. Therefore the Main Injector tuner will have to be modified for lower inductance in order to reach the required upper frequency with a gap spacing of 0.9 inches.
The lowest curve in Figure 3 illustrates the lossiness of G10 disks, which were initially mounted as supports at the location of the ceramic windows. Note these G10 disks were installed during the measurements of Figure 2.

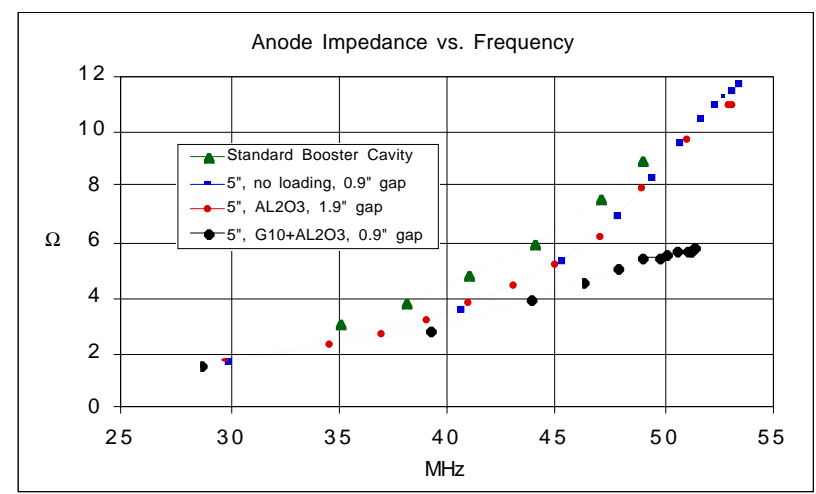

Figure 3. Curves of prototype cavity anode impedance measured using 2500 A bias supply.

\section{GRADIENT IMPROVEMENT}

There are several ways to realize higher accelerating voltage. One technique is to simply install more cavities. There exist enough Booster cavity components to install a total of 21 modified cavities in the Proton Driver, and sufficient $\mathrm{rf}$ space exists in the lattice to accommodate these cavities. Twenty-one cavities operating at the present voltage of $55 \mathrm{kV}$ per cavity would yield a ring voltage of $1155 \mathrm{kV}$. This is not far from $1188 \mathrm{kV}$, the voltage produced by 18 cavities operating at $66 \mathrm{kV}$, a $20 \%$ increase. The second way to achieve higher voltage is to raise the anode voltage on the power tubes to $30 \mathrm{kV}$. This technique has been explored on the test station where the maximum anode voltage is approximately $28 \mathrm{kV}$, limited by the high voltage power supply. The third way to higher voltage is to decrease the capacitance of the anode blocking capacitor from $1200 \mathrm{pF}$ to $400 \mathrm{pF}$, thereby increasing the step-up ratio of the cavity by about $20 \%$. A low power prototype $400 \mathrm{pF}$ capacitor has been fabricated and installed on a standard Booster cavity, and an increased step up ratio was achieved as shown in Figure 4. It is planned to modify an existing $1200 \mathrm{pF}$ blocking capacitor in a way that will allow for high power testing in the future. One caveat is that this scheme results in a $30-40 \%$ reduction of the anode impedance presented to the power amplier.

In any case, operating the cavity at higher gap voltage may be problematic due to the voltage breakdown limit in two locations: the tuner hub region and the region between the anode blocking capacitor and the outer shell of the cavity. Both locations are prone to sparking at greater than $55 \mathrm{kV}$ per cavity operation. The probability of arcing at the tuner hub decreases with frequency because the tuner impedance decreases with frequency. One technique to lower the voltage at the tuner hub is to increase the stem length thereby dropping more voltage 
across the added inductance. This would require a somewhat different tuning inductance with which to achieve the desired frequency range of 37 to $53 \mathrm{MHz}$. The arcing between the blocking capacitor and the cavity shell might be alleviated by redesigning this region for more clearance.

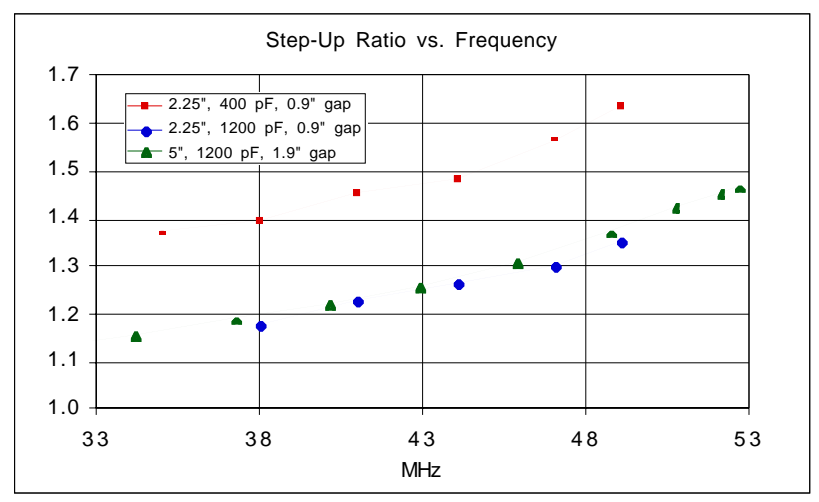

Figure 4. Booster cavity anode to gap step-up ratio with $400 \mathrm{pF}$ and $1200 \mathrm{pF}$ blocking capacitor.

\section{HIGH POWER TEST RESULTS}

The prototype cavity has been tested up to the maximum rf power capability of the test stand (Figure 5). The accelerating gaps were in air during high power testing; no ceramic windows were installed. The maximum output voltage of the high voltage power supply is about $28 \mathrm{kV}$, which limits the rf voltage at the cavity to about $32-38 \mathrm{kV}$ per gap $(64-76 \mathrm{kV}$ per cavity) over the frequency range $37-53 \mathrm{MHz}$. The cavity has been tested in two modes: single frequency and swept frequency. In swept frequency mode the cavity is operated using the usual Booster curves for anode voltage, tuner bias current, rf drive, and frequency.

Two types of Main Injector ferrite tuners exist. In one case there is a gradual change in diameter of the inner conductor near the tuner hub; in the other case there is an abrupt change in diameter. This abrupt change sufficiently enhances the electric fields so that arcing occurs near the tuner hub during high voltage operation on the prototype cavity. The tuner having the gradual change in diameter performs without arcing up to full voltage.

\section{STATUS AND OUTLOOK}

The modifications and measurements performed to date indicate the aperture enlargement goal is achievable. The next step in this effort is to convert the prototype cavity into a tunnel-ready model that can be tested at high power. Mechanical design, material requisitions, and technical drawings are in progress. Aluminum oxide ceramic windows have been ordered and are due in August. Completion of the tunnel-ready cavity is expected in Autumn, at which point high power rf testing will be performed. The higher order modes must be measured and suitable dampers must be designed, fabricated and tested prior to installing the cavity in the Booster for beam tests in the winter of 2001-2002.

\section{REFERENCES}

[1] W. Chou et al, "The Proton Driver Design Study," Fermilab TM-2136, December 2000, http://wwwlib.fnal.gov/archive/2001/tm/TM-2136.html.

[2] W. Chou et al, "A Joint Proposal for Booster Cavity Modification," Fermilab, January 12, 2000.

[3] J. E. Griffin, "Proposal to Rebuild Damaged Booster RF Cavity with Significant Improvement in Aperture, Gap Voltage, and Power Delivery Capability," Draft, November 22, 1999.

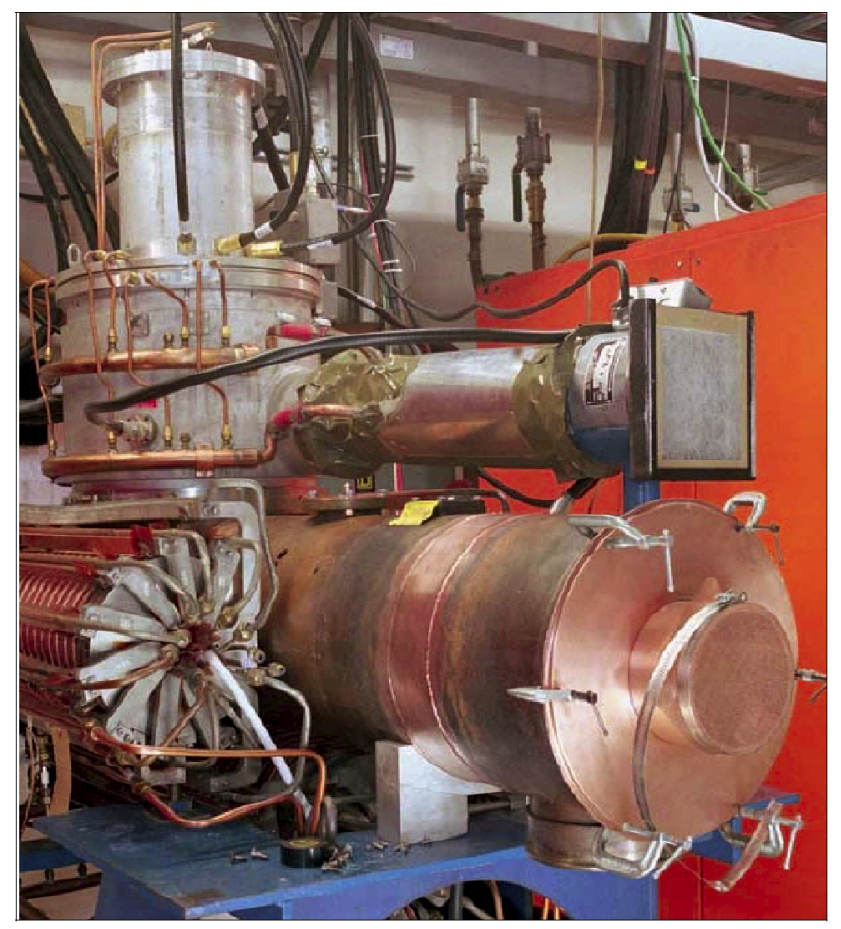

Figure 5. Photograph of the prototype large-aperture cavity with power amplifier at the test stand. 